\title{
LATTICE PROPERTIES OF THE SYMMETRIC WEAKLY INVERSE SEMIGROUP ON A TOTALLY ORDERED SET
}

\author{
C. C. EDWARDS and MARLOW ANDERSON
}

(Received 3 June 1980)

Communicated by T. E. Hall

\begin{abstract}
Let $T$ be a totally ordered set, $P_{T}$ the semigroup of partial transformations on $T$, and $A(T)$ the $l$-group of order-preserving permutations of $T$. We show that $\boldsymbol{P}_{T}$ is a regular left $l$-semigroup. Let $\boldsymbol{P}_{\boldsymbol{T}}{ }^{\prime}$ be the set of $\alpha \in P_{T}$ such that $\alpha$ is order-preserving and the domain of $\alpha$ is a final segment of $T$. Then $\boldsymbol{P}_{T}{ }^{\prime}$ is an $l$-semigroup, and we prove that it is the largest transitive $l$-subsemigroup of $\boldsymbol{P}_{T}$ which contains $A(T)$. When $T$ is Dedekind complete, we characterize the largest regular $l$-subsemigroup of $P_{T}^{\prime}$. When $A(T)$ is also $o-2$ transitive we show that there can be no $l$-subsemigroup of $P_{T}^{\prime}$ properly containing $A(T)$ which is either inverse or a union of groups.
\end{abstract}

1980 Mathematics subject classification (Amer. Math. Soc.): 20 M 20, 06 F 05.

In semigroup theory, we shall follow the terminology and notation of Clifford and Preston (1961). For a semigroup $S, E(S)$ shall denote the set of idempotents of $S$. And for $a \in S$, we shall denote an arbitrary inverse of $a$ by $a^{\prime}$ and the set of all inverses of $a$ by $V(a)$.

For an arbitrary set $T$, the symmetric weakly inverse [symmetric inverse] semigroup $P_{T}\left[I_{T}\right]$ is defined to be the set of all partial [partial one-to-one] transformations of $T$ together with the empty transformation 0 . For $\alpha \in P_{T}$, we shall denote the domain and range of $\alpha$ by $\Delta(\alpha)$ and $\nabla(\alpha)$ respectively. And we shall denote the identity map on a subset $X$ of $T$ by $1_{X}$. The following useful lemma is due to B. R. Srinivasan (1968).

LEMMA 1. $P_{T}$ is a regular semigroup and $I_{T}$ is the largest inverse subsemigroup of $P_{T}$ whose idempotents are one-to-one. Moreover, for each $\alpha \in P_{T}$,

(i) $\alpha \in E\left(P_{T}\right)$ if and only if $\nabla(\alpha) \subseteq \Delta(\alpha)$ and $\left.\alpha\right|_{\nabla(\alpha)}=1_{\nabla(\alpha)}$;

(ii) $\alpha^{\prime} \in V(\alpha)$ if and only if $\nabla\left(\alpha^{\prime}\right) \subseteq \Delta(\alpha), \nabla(\alpha) \subseteq \Delta\left(\alpha^{\prime}\right),\left.\alpha^{\prime} \alpha\right|_{\nabla(\alpha)}=1_{\nabla(\alpha)}$, and $\left.\alpha \alpha^{\prime}\right|_{\nabla\left(\alpha^{\prime}\right)}=1_{\nabla\left(\alpha^{\prime}\right)}$

OCopyright Australian Mathematical Society 1981 
In the theory of lattice-ordered groups (henceforth l-groups) and partially ordered algebraic systems we shall follow the terminology of Conrad (1970), Bigard, Keimel and Wolfenstein (1977) and Fuchs (1966). We make explicit the following definitions, not all of which are standard.

DEFINITIONS. Let $S$ be a semigroup equipped with a partial order $<$ and let $a$, $b, x$ denote arbitrary elements of $S$.

(1) $S$ is a left po-semigroup if $x a \leqslant x b$ whenever $a \leqslant b$.

(2) $S$ is a left $m$-semigroup if it satisfies (1) and in addition $(S,<)$ is a meet semilattice with $x(a \wedge b)=x a \wedge x b$.

(3) $S$ is a left $l$-semigroup if it satisfies (2) and in addition $(S,<)$ is a join semilattice with $x(a \vee b)=x a \vee x b$.

Obvious modifications of the definitions above occur when 'left' is replaced by 'right'. If a semigroup satisfies both the left and the right property, the adjective will be omitted.

Henceforth, $T$ shall denote a set equipped with a total order $<$. Let $A(T)$ be the group of all order-preserving permutations of $T$. When a partial order $<$ is defined on $A(T)$ by letting $\alpha \leqslant \beta$ if and only if $x \alpha \leqslant x \beta$ for all $x \in T, A(T)$ becomes an $l$-group. For terminology and results for this $l$-group, see Glass (1976).

In order to investigate the lattice properties which $P_{T}$ inherits from $T$ we make the following analogous definition: for $\alpha, \beta \in P_{T}, \alpha \leqslant \beta$ if and only if $\Delta(\alpha) \subseteq$ $\Delta(\beta)$ and $x \alpha \leqslant x \beta$ for all $x \in \Delta(\alpha)$. The reader may verify that this makes $P_{T}$ a lattice with the least upper bound and greatest lower bound for $\alpha, \beta \in P_{T}$ as follows. For all $x \in \Delta(\alpha \wedge \beta)=\Delta(\alpha) \cap \Delta(\beta)$,

$$
x(\alpha \wedge \beta)=x \alpha \wedge x \beta
$$

and for all $x \in \Delta(\alpha \vee \beta)=\Delta(\alpha) \cup \Delta(\beta)$,

$$
x(\alpha \vee \beta)= \begin{cases}x \alpha & x \in \Delta(\alpha) \backslash \Delta(\beta), \\ x \beta & x \in \Delta(\beta) \backslash \Delta(\alpha), \\ x \alpha \vee x \beta & x \in \Delta(\alpha) \cap \Delta(\beta) .\end{cases}
$$

We then easily obtain the following:

TheOREM 2. $P_{T}$ is a regular left l-semigroup.

When $|T|>2, P_{T}$ fails to be a right po-semigroup, and hence is not an $l$-semigroup. For if $a, b \in T$ with $a<b$, define $\alpha$ and $\beta$ by $\Delta(\alpha)=\Delta(\beta)=\{a\}$, $a \alpha=b$ and $a \beta=a$. Then $\beta<\alpha$, but $\beta^{2}=\beta \nless \alpha \beta=0$. 
Now suppose that $\alpha \leqslant \beta$ where $\alpha, \beta \in P_{T}$. What conditions on $\gamma$ will imply that $\alpha \gamma<\beta \gamma$ ? First, one needs that $\Delta(\alpha \gamma) \subseteq \Delta(\beta \gamma)$. This can be achieved if $\Delta(\gamma)$ is a final segment, that is, if $x \in \Delta(\gamma)$ and $y \geqslant x$, then $y \in \Delta(\gamma)$. Secondly, in order that $x \alpha \gamma \leqslant x \beta \gamma$ for all $x \in \Delta(\alpha \gamma)$, one clearly needs that $\gamma$ be order-preserving. Hence we are motivated to study the following subsets of $P_{T}$ :

$$
P_{T}^{\prime}=\left\{\alpha \in P_{T}: \alpha \text { is order-preserving and } \Delta(\alpha) \text { is a final segment }\right\},
$$

and

$$
S(T)=\left\{\alpha \in P_{T}^{\prime}: \Delta(\alpha)=T\right\}
$$

TheOReM 3. $P_{T}^{\prime}$ and $S(T)$ are l-semigroups.

Proof. Let $\alpha, \beta \in P_{T}{ }^{\prime}$. Clearly $\alpha \beta$ is order-preserving. To show that $\alpha \beta \in$ $P_{T}^{\prime}$, let $x \in \Delta(\alpha \beta)$ and $x \leqslant y$. Since $x \in \Delta(\alpha)$ and $\Delta(\alpha)$ is a final segment, $y \in \Delta(\alpha)$. So $x \alpha \leqslant y \alpha$ since $\alpha$ is order-preserving. But $x \alpha \in \Delta(\beta)$ and $\Delta(\beta)$ is a final segment. Hence $y \alpha \in \Delta(\beta)$, and thus $y \in \Delta(\alpha \beta)$. Consequently $P_{T}^{\prime}$ is a left po-subsemigroup of $P_{T}$. Clearly, $P_{T}^{\prime}$ is also a right po-semigroup, and thus a po-semigroup. It is obvious that when $X$ and $Y$ are final segments of $T$, we have either $X \subseteq Y$ or $Y \subseteq X$. Using this fact, the remainder of the proof is standard.

That $P_{T}^{\prime}$ is the $l$-subsemigroup of $P_{T}$ on which we should concentrate our attentions is revealed by the following theorem. Recall that a set $S$ of partial transformations of a set $T$ is transitive if for all $s, t \in T$, there exists $\alpha \in S$ with $s \alpha=t$. Obviously, $P_{T}^{\prime}$ is transitive on $T$.

THEOREM 4. $P_{T}^{\prime}$ is the largest transitive -subsemigroup of $\boldsymbol{P}_{T}$ which contains the identity.

Proof. Let $S$ be a transitive $l$-subsemigroup of $P_{T}$ containing the identity. Let $\gamma \in S$ and suppose that $x, y \in \Delta(\gamma)$ with $x<y$. Choose $\beta \in S$ with $y \beta=x$. We may assume that $\beta<1_{T}$ (replacing $\beta$ with $\beta \wedge 1_{T}$ if necessary). Then $\beta \gamma<1_{T} \gamma=\gamma$, and hence $x \gamma=y \beta \gamma \leqslant y 1_{T} \gamma=y \gamma$. So $\gamma$ is order-preserving. To show that $\Delta(\gamma)$ is a final segment, assume that $a \in \Delta(\gamma)$ and $a<b$. Choose $\alpha \in S$ with $a \alpha=b$. We may assume that $1_{T}<\alpha$, and thus $1_{T} \gamma<\alpha \gamma$. Then $\Delta\left(1_{T} \gamma\right) \subseteq \Delta(\alpha \gamma)$. Since $a \in \Delta\left(1_{T} \gamma\right)$, we must have $b=a \alpha \in \Delta(\gamma)$. It now follows that $S \subseteq P_{T}^{\prime}$.

Now, Holland's theorem (Holland (1963)) states that every $l$-group may be $l$-embedded into $A(T)$, for some totally ordered set $T$. Also, $P_{T}$ has played an 
important role in the representation theory of regular semigroups (see Munn (1970), Edwards (1979), Nambooripad (1973), and Clifford (1975)). Since $P_{T}^{\prime}$ is the largest transitive $l$-subsemigroup of $P_{T}$ containing $A(T), P_{T}^{\prime}$ could play an important role in a representation theory for regular $l$-semigroups. There are difficulties with this conjecture, however, as Theorems 10 and 12 of this paper will reveal.

In fact, the $l$-semigroup $P_{T}^{\prime}$ need not be regular; as an example, take $T=\mathbf{R}=\Delta(\alpha)$, and $\alpha=$ arctangent. Consequently, we are naturally led to ask: are there any regular $l$-subsemigroups of $P_{T}{ }^{\prime}$ ?

Consider first the special case $I_{T} \subseteq P_{T}$, where $I_{T}{ }^{\prime}=P_{T}{ }^{\prime} \cap I_{T}$. If $\alpha \in I_{T}$, then $\Delta\left(\alpha^{-1}\right)=\nabla(\alpha)$, and so a regular (and hence inverse) subsemigroup of $I_{T}{ }^{\prime}$ must have the added property that the range of each element is also a final segment. However, these considerations lead to the following results, stated without proof:

Proposition 5. If $C=\left\{\alpha \in P_{T}^{\prime}: \nabla(\alpha)\right.$ is a final segment $\}$, then $C$ is an m-semigroup but not an l-semigroup. Moreover, if $S$ is an l-subsemigroup of $C$ containing the one-to-one idempotents in $C$, then $S$ is a band.

However, we are able to identify the largest regular $l$-subsemigroup of $P_{T}{ }^{\prime}$ when $T$ is Dedekind complete (see Theorem 7). When $T$ is not Dedekind complete, many more elements of $P_{T}^{\prime}$ fail to have inverses. For example, let $T=\mathbf{Q}$ and define $\gamma$ by $x \gamma=x$ when $x<\pi$ and $x \gamma=x+1$ when $x>\pi$. Then $\gamma$ has no inverse in $P_{\mathbf{Q}}$. However, any extension $\bar{\gamma}$ of $\gamma$ in $P_{\mathbf{R}}^{\prime}$ does admit a unique inverse in $\boldsymbol{P}_{\mathbf{R}}{ }^{\prime}$.

Now, if $T$ is totally ordered and $T$ is its Dedekind completion, then $A(T)$ may be $l$-embeded into $A(T)$ by a map ${ }^{-}$so that for all $\alpha \in A(T), \bar{\alpha} \mid T=\alpha$ (see Glass (1976), p. 32). And certainly for each $\alpha \in P_{T}^{\prime}$ there exist (perhaps many) $\bar{\alpha} \in P_{T^{\prime}}$ such that $\bar{\alpha} \mid T=\alpha$. However, it is impossible in general to define such a function so that it is a semigroup homomorphism, as the following theorem shows.

TheOREM 6. There exists no semigroup homomorphism 'embedding $\boldsymbol{P}_{\mathbf{Q}}$ ' into $\boldsymbol{P}_{\mathbf{R}}{ }^{\prime}$ such that $\bar{\alpha} \mid \mathbf{Q}=\alpha$ for all $\alpha \in P_{\mathbf{Q}}^{\prime}$. In particular, $S(\mathbf{Q})$ cannot be embedded into $S(\mathbf{R})$.

Proof. Assume to the contrary that such a semigroup homomorphism ${ }^{-}$exists. There exists an order isomorphism $\tau$ from $\mathbf{Q} \cap(-\infty, \pi)$ onto $\mathbf{Q} \cap(-\infty, 0)$, since 
they both are countable dense totally ordered sets. Define elements $\alpha, \beta$, and $\gamma$ of $P_{\mathbf{Q}^{\prime}}$ as follows:

$$
\begin{aligned}
& x \alpha=\left\{\begin{array}{ll}
x \tau & \text { if } x<\pi, \\
x & \text { if } x>\pi ;
\end{array} \quad x \beta= \begin{cases}x & \text { if } x<0, \\
x+1 & \text { if } x>0 ;\end{cases} \right. \\
& x \gamma= \begin{cases}x & \text { if } x<\pi, \\
x+1 & \text { if } x>\pi .\end{cases}
\end{aligned}
$$

Then $\alpha \beta=\gamma \alpha=\alpha \gamma$, and so $\bar{\alpha} \bar{\beta}=\bar{\gamma} \bar{\alpha}=\bar{\alpha} \bar{\gamma}$. Thus,

$$
\pi \bar{\alpha}+1=\pi \bar{\alpha} \bar{\beta}=\pi \bar{\gamma} \bar{\alpha} \in \nabla(\bar{\alpha}) .
$$

This means that $\pi \bar{\alpha}+1>\pi$. But $\pi \bar{\alpha}+1 \in \nabla(\bar{\gamma})$, which means that $\pi \bar{\alpha}+1=$ $\pi \bar{\gamma}$ or $\pi \bar{\alpha}+1>\pi+1$. But $\pi \bar{\alpha}+1>\pi+1$ is impossible since $0<\pi \bar{\alpha}<\pi$. Consequently, $\pi \bar{\alpha} \bar{\gamma}=\pi \bar{\alpha}+1=\pi \bar{\gamma}$. Since $\bar{\gamma}$ is one-to-one, $\pi \bar{\alpha}=\pi$, and thus $\pi \bar{\gamma}=\pi+1$.

Now choose an order isomorphism $\sigma$ from $Q \cap(\pi, \infty)$ onto $Q \cap(6, \infty)$, and consider elements $\xi, \theta$, and $\eta$ of $\boldsymbol{P}_{\mathbf{Q}}{ }^{\prime}$ defined as follows:

$$
\begin{aligned}
& x \xi=\left\{\begin{array}{ll}
x & \text { if } x<\pi, \\
x \sigma & \text { if } x>\pi ;
\end{array} \quad x \theta= \begin{cases}x-1 & \text { if } x<6, \\
x & \text { if } x>6 ;\end{cases} \right. \\
& x \eta= \begin{cases}x-1 & \text { if } x<\pi, \\
x & \text { if } x>\pi .\end{cases}
\end{aligned}
$$

An argument similar to the one above results in $\pi \bar{\eta}=\pi-1$. Let $\nu \in A(\mathbf{Q})$ be defined by $x \nu=x+1$. Then $\eta \nu=\gamma$. And thus,

$$
\pi=(\pi-1)+1=\pi \bar{\eta} \bar{\nu}=\pi \bar{\gamma}=\pi+1,
$$

which is a contradiction. So $P_{\mathbf{Q}}{ }^{\prime}$ cannot be embedded into $P_{\mathbf{R}}$. Since all elements in this proof have $Q$ for their domain, we have also shown that $S(Q)$ cannot be embedded into $S(\mathbf{R})$.

In light of the above remarks, we shall henceforth assume that $T$ is Dedekind complete. And we shall henceforth denote $P_{T}{ }^{\prime}$ simply by $P$.

As a starting point for examining the existence of inverses of elements in $P$, we shall make a few remarks concerning $E(P)$. As before, if $\xi \in E(P)$, then $\nabla(\xi) \subseteq \Delta(\xi)$ and $\xi$ is the identity map on $\nabla(\xi)$ (see Lemma 1 ). But $\xi$ is also order-preserving. Thus if $x \xi \neq x$, then there exists an interval of $T$ containing $x$ and $x \xi$ on which $\xi$ is constant. Also, when $|T|>2$, it is not difficult to show that the idempotents of $P$ (and of $S(T)$ ) do not form a band.

We now determine those elements of $P$ which possess inverses. For $\alpha \in P$ and $x \notin \nabla(\alpha)$, let $I_{x}$ be the maximum convex subset of $T$ for which $I_{x} \subseteq T \backslash \nabla(\alpha)$ and $x \in I_{x}$. Since $I_{x}$ is an interval of some configuration, we need to establish some notational conventions for interval notation. First of all, $(-\infty, a)$ and 
$(a, \infty)$ shall denote the maximum initial and final intervals excluding $a$. Secondly, we shall restrict the use of square brackets, so that $[a, b)$, for example, shall mean not only $\{x: a \leqslant x<b\}$, but also that

$$
a=\bigvee\{x \in T: x<a\} \text {. }
$$

The analogous restriction applies to upper endpoints. With these conventions in force, we now observe that $I_{x}=[a, b]$ (with $a$ and $b$ possibly equal) is impossible. For suppose the contrary. Let $\mathcal{L}$ denote the smallest initial segment containing $(-\infty, a) \alpha^{-1}$ and let $U=(a, \infty) \alpha^{-1}$. Since $U$ is a final segment, $\mathfrak{L} \cup \mathcal{U}=T$ and $\mathcal{L}<\mathcal{U}$. Since $T$ is Dedekind complete we have $\vee \mathfrak{E}=\wedge \mathscr{U}$ $=t \in T$. But by definition of $I_{x}$, we have that $a=\bigvee\{x \alpha: x \alpha<a\}$ and $b=\wedge\{x \alpha: x \alpha>b\}$. Now $t \alpha=(\bigvee \mathfrak{L}) \alpha \geqslant x \alpha$, for all $x \alpha<a$, and so $t \alpha>a$. Similarly $t \alpha \leqslant b$. Thus, $t \alpha=a=b \in \nabla(\alpha)$, a contradiction.

We are now able to distinguish those elements of $P$ (and of $S(T)$ ) which have inverses. Let

$$
R=\{\alpha \in P: \bigvee \nabla(\alpha), \text { if it exists, is in } \nabla(\alpha)\}
$$

and

$$
R(T)=\{\alpha \in S(T) \cap R: \wedge \nabla(\alpha), \text { if it exists, is in } \nabla(\alpha)\} .
$$

THEOREM 7. $R$ is the maximum regular subsemigroup of $P$; in fact it is an l-subsemigroup of $P$.

Proof. We first show that any element $\alpha \in P \backslash R$ possesses no inverse in $P$. That $\alpha \notin R$ means that some $I_{x}$ is of the form $[a, \infty)$. Suppose $\alpha^{\prime}$ is an inverse of $\alpha$ in $P$. Now because $\Delta\left(\alpha^{\prime}\right) \supseteq \nabla(\alpha)$ and $\Delta\left(\alpha^{\prime}\right)$ is a final segment, $a \in \Delta\left(\alpha^{\prime}\right)$. And since $a>\nabla(\alpha)$ and $\alpha^{\prime} \alpha$ is the identity map on $\nabla(\alpha)$ (see Lemma 1), $a \alpha^{\prime} \alpha>\nabla(\alpha)$. But because $a \alpha^{\prime} \alpha \in \nabla(\alpha)$, this means that $\nabla(\alpha)$ has a greatest element, which is a contradiction.

We will now show that each element of $R$ possesses an inverse. Let $\alpha \in R$. To define an inverse $\alpha^{\prime}$, we first specify that $\Delta\left(\alpha^{\prime}\right)$ shall be the smallest final segment of $T$ which contains $\nabla(\alpha)$. For each preimage set $x \alpha^{-1}$, choose a fixed $y_{x} \in$ $x \alpha^{-1}$. For $x \in \Delta\left(\alpha^{\prime}\right)$, define $x \alpha^{\prime}$ as follows:

$$
x \alpha^{\prime}= \begin{cases}y_{x} & \text { if } x \in \nabla(\alpha), \\ y_{a} & \text { if } I_{x}=(a, b),(a, b] \text { or }(a, \infty), \\ y_{b} & \text { if } I_{x}=[a, b) .\end{cases}
$$

Note that $\alpha^{\prime}$ is defined for all $x \in \Delta\left(\alpha^{\prime}\right)$, because $I_{x}=[a, b]$ or $[a, \infty)$ is impossible, and $I_{x}=(-\infty, b]$ or $(-\infty, b)$ makes $x$ not an element of $\Delta\left(\alpha^{\prime}\right)$. It is obvious that $\alpha \alpha^{\prime} \alpha=\alpha$, and easily verified that $\alpha^{\prime} \in P$. So $\alpha^{\prime} \alpha \alpha^{\prime} \in V(\alpha) \cap P$, and thus also in $R$. Finally, a little calculation is sufficient to show that $R$ is an l-subsemigroup of $P$. 
Putting together Theorems 5 and 7 yields:

COROLlaRY 8. $R$ is the maximum transitive regular l-subsemigroup of $P_{T}$ which contains $A(T)$.

A minor modification of the proof above (just let $x \alpha^{\prime}=b \alpha^{\prime}$ if $I_{x}=(-\infty, b)$ ) yields:

COROLlaRy 9. $R(T)$ is the maximum regular subsemigroup of $S(T)$; it is in fact an l-subsemigroup of $S(T)$.

We shall now turn to the question of inverse $l$-subsemigroups of $P$ which contain $A(T)$. If $A(T)$ is $o-2$ transitive (that is, if whenever $s, t, u, v \in T$ with $s<t$ and $u<v$, then there exists $\alpha \in A(T)$ with $s \alpha=u$ and $t \alpha=v)$, we shall prove that no such semigroups other than $A(T)$ exist. We need the following lemma.

LEMMA. Let $S$ be a subsemigroup of $P$ which contains an $o-2$ transitive subset of $A(T)$ and an idempotent $\xi$ whose range is not convex. Then $S$ contains an idempotent $\eta$ which does not commute with $\xi$. (Hence $S$ is not inverse.)

Proof. Suppose $\xi$ is an idempotent of $S$ whose range is not convex; then there exists a point $d \in \Delta(\xi)$ where $\{t \in \Delta(\xi): t<d\}$ and $\{t \in \Delta(\xi): t>d\}$ are nonempty, and $\xi$ is not continuous at $d$. The following cases cover all possibilities (although they are not disjoint from one another):

Case (i): $d \xi=d$ and $x \xi=r>d$ for all $d<x<r$.

Choose $k \in T$ with $d<k<r$ (because $A(T)$ is $o-2$ transitive, $T$ is dense in itself (Glass (1976))). Let $\tau \in S \cap A(T)$ be such that $d \tau=k$ and $k \tau=r$. Then $\eta=\tau \xi \tau^{-1} \in S$ and it is idempotent. But

$$
\begin{aligned}
d \xi \eta & =d \tau \xi \tau^{-1}=k \xi \tau^{-1}=r \tau^{-1}=k<r \\
& =k \xi=r \tau^{-1} \xi=k \xi \tau^{-1} \xi=d \eta \xi .
\end{aligned}
$$

Case (ii): $d \xi=d$ and $x \xi=s<d$ for all $s<x<d$.

This is entirely similar to Case (i).

Case (iii): $d \xi>d$.

Choose $k \in \Delta(\xi)$ with $k<d$. Then $k \xi \leqslant d$. Pick $\tau \in S \cap A(T)$ such that $d \xi \tau=d$ and $d \tau=k$. Then $\eta=\tau \xi \tau^{-1}$ is idempotent and

$$
\begin{aligned}
d \xi \eta & =d \xi \tau^{-1}>d \tau^{-1}=d \xi=d \xi^{2} \\
& =d \tau^{-1} \xi>k \xi \tau^{-1} \xi=d \eta \xi .
\end{aligned}
$$


Case (iv): $d \xi<d$.

This is similar to Case (iii).

Let

$$
N=\left\{\begin{array}{l}
\alpha \in R: \nabla(\alpha) \text { is convex, and every maximal interval } \\
\text { on which } \alpha \text { is constant is final and/or initial in } \Delta(\alpha)
\end{array}\right\} .
$$

Also, let $N(T)=R(T) \cap N$.

TheOREM 10. Suppose that $A(T)$ is o- 2 transitive. Then $N$ is the maximum inverse subsemigroup of $P$ which contains $A(T)$. There exist no inverse l-subsemigroups of $P$ which properly contain $A(T)$.

Proof. It is easily verified that $N$ is an inverse subsemigroup of $P$. If $\alpha \in R \backslash N$ and $\alpha^{\prime}$ is any inverse of $\alpha$, we shall show that either $\alpha \alpha^{\prime}$ or $\alpha^{\prime} \alpha$ is an idempotent whose range is not convex. It will then follow from the lemma that there can exist no inverse subsemigroup of $P$ which contains $\alpha$ and $A(T)$.

Suppose $\alpha \notin N$. If $\nabla(\alpha)$ is not convex, then $\nabla\left(\alpha^{\prime} \alpha\right)=\nabla(\alpha)$ is not convex. If $\alpha$ is constant on the maximal interval $[a, b]$ which is neither initial nor final, then for each inverse $\alpha^{\prime}$ of $\alpha$ there exists $k \in[a, b]$ such that $k \notin \nabla\left(\alpha^{\prime}\right)$. But then $\nabla\left(\alpha \alpha^{\prime}\right)=\nabla\left(\alpha^{\prime}\right)$ is not convex.

Now suppose that $S$ is an inverse $l$-semigroup properly containing $A(T)$; then $S$ contains an idempotent $\xi$, not equal to the identity $1_{T}$. Because $S \subseteq N, \xi$ is of the following form:

$$
x \xi= \begin{cases}a, & x<a, \\ x, & a<x<b, \\ b, & b<x,\end{cases}
$$

(where $a=-\infty, b=\infty, a=b$ are all possible). By taking $\xi \vee 1_{T}$ or $\xi \wedge 1_{T}$, we may assume that $\xi$ is of one of these two forms:

$$
x \xi= \begin{cases}x, & x<a \\ a, & x>a\end{cases}
$$

where there exists $b<a$ with $b \in \Delta(\xi)$; or

$$
x \xi= \begin{cases}a, & x<a \\ x, & x>a\end{cases}
$$

where there exists $b<a$ with $b \in \Delta(\xi)$.

We shall henceforth assume $\xi$ is of the first form, since a straightforward modification of the proof below will handle the second case. So, choose 
$b, c \in \Delta(\xi)$ with $c<b<a$. Also pick $\sigma, \tau \geqslant 1_{T}$ in $A(T)$ with $c \tau=a$ and $c \boldsymbol{\sigma}=b$. Then

$$
x \tau \xi \tau^{-1} \sigma= \begin{cases}x \sigma, & x \leqslant c \text { with } x \tau \in \Delta(\xi) \\ b, & x \geqslant c\end{cases}
$$

Consequently,

$$
x\left(\tau \xi \tau^{-1} \sigma \vee \xi\right)= \begin{cases}x \sigma, & x \leqslant c \text { with } x \tau \in \Delta(\xi), \\ b, & c \leqslant x \leqslant b, \\ x, & b \leqslant x \leqslant a, \\ a, & a \leqslant x .\end{cases}
$$

But $\tau \xi \tau^{-1} \sigma \vee \xi$ is then not an element of $N$, which is a contradiction.

Corollary 11. Suppose that $A(T)$ is o-2 transitive. Then $N(T)$ is the maximum inverse subsemigroup of $S(T)$ which contains $A(T)$. There exist no inverse l-subsemigroups of $S(T)$ which properly contain $A(T)$.

In a similar fashion, we show that $l$-subsemigroups properly containing $A(T)$ which are unions of groups do not exist (for a discussion of $l$-semigroups which are unions of groups, see Anderson and Edwards (1980)).

TheORem 12. Suppose that $A(T)$ is $o-2$ transitive. Then there exist no l-subsemigroups of $P$ properly containing $A(T)$ which are unions of groups.

Proof. Let $S$ be an $l$-subsemigroup of $P$ which properly contains $A(T)$, and suppose $S$ is a union of groups. Let $\xi$ be a non-identity idempotent of $S$. Then there exists an interval $[a, b]$ with $a<b$ on which $\xi$ is constant, and with $a \leqslant a \xi \leqslant b$. Suppose that $a \xi<b$. Then choose $c_{1}, c_{2}, c_{3}$ and $c_{4}$ with $a \xi<c_{1}<c_{2}$ $<c_{3}<c_{4}<b$. Since $A(T)$ is $o-2$ transitive, it is $o-3$ transitive (see Glass (1976), p. 39) and so there exists $\tau \in A(T)$ with $c_{3} \tau=a \xi, c_{4} \tau=c_{1}, b \tau=c_{2}$. And since $S$ is a union of groups, $\xi \vee \tau$ is contained in some $\mathcal{H}$-class $H$ which is a maximal group (see Clifford and Preston (1961), pp. 48, 61). Let $\beta$ be the inverse of $\xi \vee \tau$ in $H$. Now $\xi \vee \tau$ is constant on $\left[a \xi, c_{3}\right]$ and so $\nabla(\beta) \cap\left(b \xi, c_{3}\right)$ contains at most one point. But because $\beta$ and $\xi \vee \tau$ are $\mathcal{C}$-related, we have that $\nabla(\beta)=\nabla(\xi \vee \tau)$ (Clifford and Preston (1961), Lemma 2.5) and $c_{1}, c_{2} \in$ $\nabla(\xi \vee \tau) \cap\left(b \xi, c_{3}\right)$, which is a contradiction. If $a<a \xi$, a similar argument involving the infimum of $\xi$ with an element of $A(T)$ leads to the same contradiction. Thus, no $l$-subsemigroup of $P$ properly containing $A(T)$ is a union of groups. 
There are many avenues of further research suggested by the results of this paper. We shall conclude by mentioning a few of them. The general question remains: does there exist an analogue to Holland's theorem for $l$-semigroups making use of $P_{T}{ }^{\prime}$ ? More concretely, we can ask this: what $l$-semigroups are representable as $l$-subsemigroups of $P_{T}^{\prime}$, for some totally ordered set $T$ ? A question perhaps related to this is the following: give an abstract characterization of semigroups of the form $\boldsymbol{P}_{T}{ }^{\prime}$. Finally, the proof of Holland's theorem suggests another line of inquiry. What is the structure of the lattice of $l$-congruences and the lattice of left l-congruences on $P_{T}^{\prime}$, and on an arbitrary $l$-semigroup? Much of the structure of $l$-groups is describable in terms of these lattices (see Conrad (1970)) and so as much should be expected for $l$-semigroups.

\section{References}

M. E. Anderson and C. C. Edwards (1980), 'Unions of l-groups', to appear.

A. Bigard, K. Keimel and S. Wolfenstein (1977), Groupes et anneawx reticules (Springer-Verlag, Berlin).

A. H. Clifford (1975), 'The fundamental representation of a regular semigroup', Semigroup Forum 10, 84-92.

A. H. Clifford and G. B. Preston (1961), The algebraic theory of semigroups (American Math. Soc., Providence).

P. Conrad (1970), Lattice-ordered groups (Tulane Library, New Orleans).

C. C. Edwards (1979), 'The structure of $\mathcal{L}$-unipotent semigroups', Semigroup Forum 18, 189-199.

L. Fuchs (1966), Teilweise geordnete algebraische Strukturen (Vandenhoeck and Ruprecht, Gottingen).

A. M. W. Glass (1976), Ordered permutation groups (Bowling Green State University, Bowling Green, Ohio).

W. C. Holland (1963), 'The lattice-ordered group of automorphisms of an ordered set', Michigan Math. J. 10, 399-408.

W. D. Munn (1970), 'Fundamental inverse semigroups', Quart. J. Math. Oxford Ser. 21, 157-170.

K. S. S. Nambooripad (1973), Structure of regular semigroups (Dissertation, Univ. of Kerala, India).

B. R. Srinivasan (1968), 'Weakly inverse semigroups', Math. Ann. 176, 324-333.

Department of Mathematical Sciences

Indiana University-Purdue University at Fort Wayne

Fort Wayne, Indiana 46805

U.S.A. 